\title{
ROLE OF SOIL ALGAE ON THE INITIAL STAGES OF SOIL FORMATION IN SANDY POLLUTED AREAS
}

\author{
ROLA GLONÓW GLEBOWYCH W INICJALNYCH ETAPACH ROZWOJU \\ GLEBY NA ZANIECZYSZCZONYCH OBSZARACH PIASZCZYSTYCH
}

\begin{abstract}
Research to evaluate the role of the soil algae in the initiation of pedogenesis in sandy areas and to establish the mineral, chemical and organic composition of soil-algal crusts was done. The investigations area is located in southern Poland in a landscape of drift sand (Bledowska Desert) formed as a result of medieval deforestation related to $\mathrm{Ag}, \mathrm{Pb}$ and $\mathrm{Fe}$ mining activities. Fifteen species of algae (Cyanophyta, Chlorophyta and Heterokontophyta) were identified. In the soil-algal crusts, $\mathrm{C}_{\text {org }}$ contents ranged from $0.35-2.23 \%, \mathrm{P}_{\text {avail }}$ from 9-34 mg $\mathrm{kg}^{-1}$ and $\mathrm{N}_{\mathrm{t}}$ was variable. In investigated area the ground on which soil algae developed was acidic ( $\mathrm{pH}$ 4.4-5.7 in $\mathrm{KCl}$ ). Among mineral components in the crust, phases rich in $\mathrm{Fe}$ and $\mathrm{K}$ dominated $\left(776.5( \pm 2.3)-2803( \pm 31) \mathrm{mg} \cdot \mathrm{kg}^{-1}\right.$ and $230.5( \pm 3.7)-696( \pm 22) \mathrm{mg} \cdot \mathrm{kg}^{-1}$, respectively) and elevated concentrations of $\mathrm{Zn}, \mathrm{Mn}$ and $\mathrm{Pb}$ occurred. Soil-crust organic matter includes aliphatic and aromatic compounds, carbohydrate derivatives, phenols, furan and pyrene structures. $\mathrm{N}$ compounds have significant contribution in composition of soil-algal crusts - the algae are main source of organic matter in this stage of soil formation. It could accelerate the regeneration of disturbed ecological systems.
\end{abstract}

Keywords: heavy metals, organic compounds, soil algae, soil-algal crust, soil development

\section{Introduction}

Soil algae play an important role in all stages of soil development. At different stages, ecologically diverse group of algae participate in this processes [1,2]. Algae influence is seen most clearly at the initial stage of soil formation, ie during the colonization of an abiotic ground and the formation of a primary layer enriched in organic carbon on this mineral substrate. Almost to the present, this role has been ascribed to lichens. Autotrophic algae have an ability to produce and accumulate organic matter and, by doing so, stimulate the development of heterotrophic organisms. In the soil environment, they initiate

\footnotetext{
${ }^{1}$ Faculty of Earth Sciences, University of Silesia, ul. Będzińska 60, 41-200 Sosnowiec, Poland, phone +48323689306

${ }^{2}$ Faculty of Earth Sciences, Nicolaus Copernicus University, ul. J. Gagarina 9, 87-100 Toruń, Poland

${ }^{3}$ Faculty of Food Technology, University of Agriculture in Krakow im. Hugona Kołłątaja, ul. Balicka 122, 30-149 Kraków, Poland

${ }^{*}$ Corresponding author: oimahmad.rahmonov@us.edu.pl
} 
biochemical processes that release nitrogen and phosphorus. Potassium, calcium, magnesium and other microelements are released from mineral substances [3, 4].

Algae are very significant to soil formation in arid and semiarid regions [4] and in moderate climate zones, both in natural and anthropogenic ecosystems [5-7]. Their participation in primitive soil formation, particularly in desert areas, was thoroughly explained in different works [7, 8]. As algae, lichens and lower plants form the basic biological set in arid areas, their importance and role in soil formation is better known in those environments than it is for soils in moderate climate zones, where higher plants are much more important $[5,6]$.

The term 'soil algae' includes the whole set of ecologically diverse groups such as (a) algae growing over the entire surface of soil and forming a crust, (b) algae living on moist soil surfaces and (c) algae occurring within the soil $[9,10]$. Underground soil algae in arid habitats rarely occur as solitary organisms but are, more commonly, associated with fungi, lichens, club mosses and bryophytes forming continuous biological soil crusts [3, 9, 11]. In this work, an soil-algal crust is defined as surface algae living on grains of sand. Such surfaces have variable degrees of ground stability like structural, physical, resistance against wind and water erosion. Though algae play an important role during initial pedogenesis processes.

The taxonomy and occurrence of the soil algae has been described in only a few papers in Poland [6, 12-14]. In unstable sandy areas, organisms with higher edaphic requirements cannot survive. The first, most important stage of soil formation is the stabilization of the sandy surface by microorganisms. Algae are the first organisms to colonize areas exposed to wind erosion and metal-pollution [14-16]. The formation of soil crusts increases resistance to deflation $[7,11]$.

The special location of the investigated area gives good possibility for the recognition of the roles of algae in the soil formation under conditions of environmental pollution especially with heavy metals. Soil crusts are developed on the anthropogenic area of the sand desert (Bledowska Desert) formed on fluvioglacial sands in moderate climate of Europe. The area is located 3 kilometers from sources of mining and smelting heavy metals pollutions. In addition it overlies the direction of winds transporting the pollution from the nearby Upper Silesian Industrial District.

The aim of the work reported here was to evaluate the role of soil algae on the initial stages of soil formation in sandy regions and to determine the chemical, mineralogical and organic composition of soil-algal crusts.

\section{Materials and methods}

\section{Study area}

The research was carried out in the area of the Bledowska Desert located close to Olkusz and Krakow (Fig. 1). The investigated area is situated near the Pomorzany $\mathrm{Zn}-\mathrm{Pb}$ Mine and the Boleslaw Smelter as main sources of mineral aggregates rich in heavy metals. In the subsoil basement, there are Triassic carbonate rocks containing $\mathrm{Zn}-\mathrm{Pb}$ deposits of Mississippi Valley Type, overlaid by Pleistocene fluvioglacial sands $60-70 \mathrm{~m}$ thick [16].

Since the sub-boreal period (5100 BP), the area has been covered by mixed forest with Pinus sylvestris as the dominating species [16]. Since the early Medieval (11 ${ }^{\text {th }}$ Century), intensive mining and smelting of silver, lead and iron ores has been carried out in the 
Silesia-Krakow Region. A few centuries of sawing, left the area deforested. This resulted in the degradation mainly by wind of poorly developed covers of podzol soils and the formation of a sandy area $(5 \times 12 \mathrm{~km})$ showing the features of a desert landscape (Bledowska Desert). Several years ago, Salix acitifolia was artificially introduced into the sandy area which, aided by a spontaneous succession of $S$. arenaria and Pinus sylvestris, led to the partial overgrowth of the desert. Large deflation areas and parts of the desert not colonized by higher vegetation provide good sites for investigating changes related to soil formation processes in which soil algae play significant roles [6].

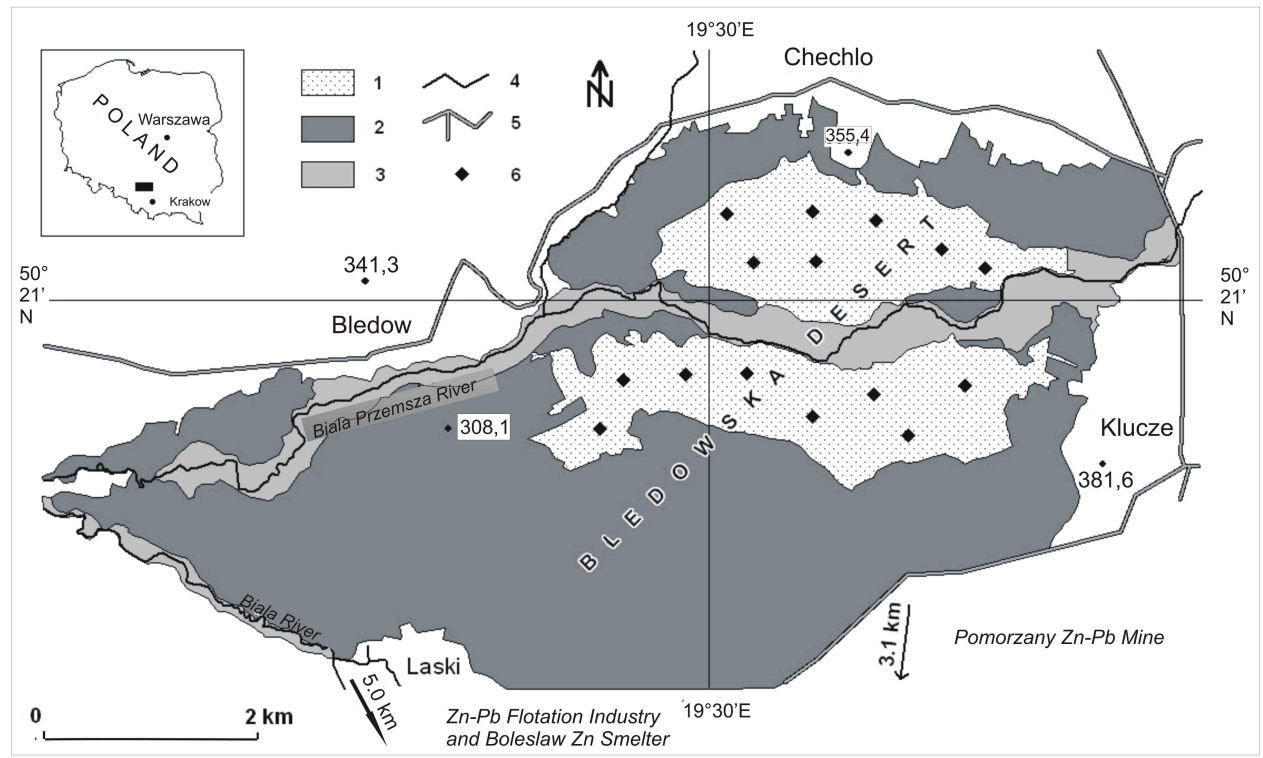

Fig. 1. The location of investigated area: 1 - sandy areas, 2 - forest cover, 3 - riverside areas, 4 - water courses, 5 - roads and 6 - sampling sites

\section{Sampling}

Sampling of soil algae was done in April and May 2010, the months with the highest humidity. The samples from 15 sampling sites (Table 1, Fig. 1) were taken to the laboratory in Petri dishes. The identification of algae and cyanobacteria species was carried out on a living material. A detailed taxonomic characterization of the species of cyanobacteria and algae recorded is presented in the other work [6] and in the base of Algae Base [17].

Soil-algal crusts occurring on the surface of the initial humus horizon (OA ectoorganic type) were sampled over an area of about $0.5 \mathrm{~m}^{2}$. Samples were stored in $1000 \mathrm{~cm}^{3}$ glass containers.

\section{Chemical analysis}

After drying at ambient temperature, and crushing/sieving and homogenization, the following parameters were measured in the soil-algal crust samples: organic carbon content $\left(\mathrm{C}_{\mathrm{org}}\right.$; Turin method $)$, total nitrogen content $\left(\mathrm{N}_{\mathrm{t}}\right.$; Kjeldahl method), available phosphorus 
(Egner-Riehm method), and $\mathrm{pH}$ (reaction with a glass electrode, in $\mathrm{H}_{2} \mathrm{O}$ and in $1 \mathrm{~mol} \cdot \mathrm{dm}^{-3}$ $\mathrm{KCl})$.

Calcium, magnesium, potassium and sodium were determined by validated Flame Atomic Absorption Spectrometry - AAS (Varian AA240FS) according to the Polish Standard PN-EN 15505: 2008 and iron, zinc and manganese according to PN-EN 14084: 2004. Determination of cadmium and lead was carried out with validated Electrothermal Atomic Absorption Spectrometry (Varian AA240Z; graphite cuvette) according to the Polish Standard PN-EN 14084: 2004. Samples were dissolved using the CEM MARSX press microwave method, using nitric acid (MERCK Suprapur; catalogue No. 1.00441; $0.01 \mathrm{dm}^{3}$ per $0.5 \mathrm{~g}$ of sample). As part of the quality control of the method, the Certified Reference Material NCS ZC 73009 (China National Analysis Center for Iron and Steel) was tested.

Each sample was analyzed in triplicate for soil chemical properties and fivefold element concentration (AAS).

\section{Pyrolitic analysis}

Among the samples tested by MS (Mass spectral) sample number 2 is the most representative (Table 1), because the soil-algal crust with Cylindrocapsa sp. dominate there. This sample came from a part of the deflation area with the greatest thickness of soil-algal crust $(6 \mathrm{~mm})$, and where the sandy material under the crust contained the highest percentage of clay fraction and organic carbon of all the 15 samples (Table 2).

\section{Analytical conditions of the Curie-point Py-GC/MS}

A Curie-point Pye-Unicam pyrolyser (type 795050) working at $770^{\circ} \mathrm{C}$ was coupled to a Hewlett-Packard gas chromatograph with a HP-5 column $(60 \mathrm{~m} \cdot 0.32 \mathrm{~mm} \cdot 0.5 \mu \mathrm{m})$. The experimental conditions were as follows: carrier gas - He at 15 psi pressure; carrier gas split ratio 1:15; temperature program: $40^{\circ} \mathrm{C}$ and final temperature $260^{\circ} \mathrm{C}$ held for $16 \mathrm{~min}$. The mass spectrometer was operated in the electron impact ionization mode at $70 \mathrm{eV}$ and scanned from 30-500 da (full scan). Data were processed using the Hewlett Packard Chemstation software. All compounds were identified by their mass spectra, comparison of retention times of their peaks to these of standard compounds, reference to literature data, and by interpretation of MS fragmentation patterns [16, 18, 19].

\section{SEM-EDXRA investigation}

Electron-probe microanalyses and observations were performed using an Environmental Scanning Electron Microscope (Philips XL 30) with an EDXRA analyzer. Pieces $(1.0 \mathrm{~cm})$ of algal crust with attached soil particles were fixed to aluminium stubs with double-sided carbon tape immediately after collection. Mineral grains attached to algal surfaces were treated as soil-algal crust in the current study. For each sample, a few hundred representative particles were placed onto a carbon adhesive base and examined using a stereo microscope. 16 samples of soil-algal crust were viewed uncoated in an SEM using a back scattered electrons (BSE) detector (Centaurus). 60 qualitative microanalyses (EDXRA) of mineral and biotic material for all the 16 samples were carried out using an attached EDXRA (Sapphire) energy dispersive X-ray spectrometer (15 kV accelerating voltage, environmental mode and $40 \mathrm{~Pa} \mathrm{H}_{2} \mathrm{O}$ pressure). $120 \mathrm{BSE}$ images of soil algal crust 
and soil material for all the 16 samples were registered to document analysed material. The presence of characteristic peaks of some elements in the EDXRA spectra enabled determination of elemental compositions.

\section{Statistical analyses}

Received results were verified by the statistical analyses. All of them were done using the Statistica 10.0 package. The significant differences between average values were estimated using the Duncan test (for $p<0.05$ ).

\section{Results}

\section{Species composition}

Algae, cyanobacteria, fungi and microorganisms are primary organisms colonizing surfaces of parent rock. Algae developing on the sand surface in the early-spring wet seasons form an easily-seen green coat which vanishes in seasons of water deficiency at the beginning of the summer.

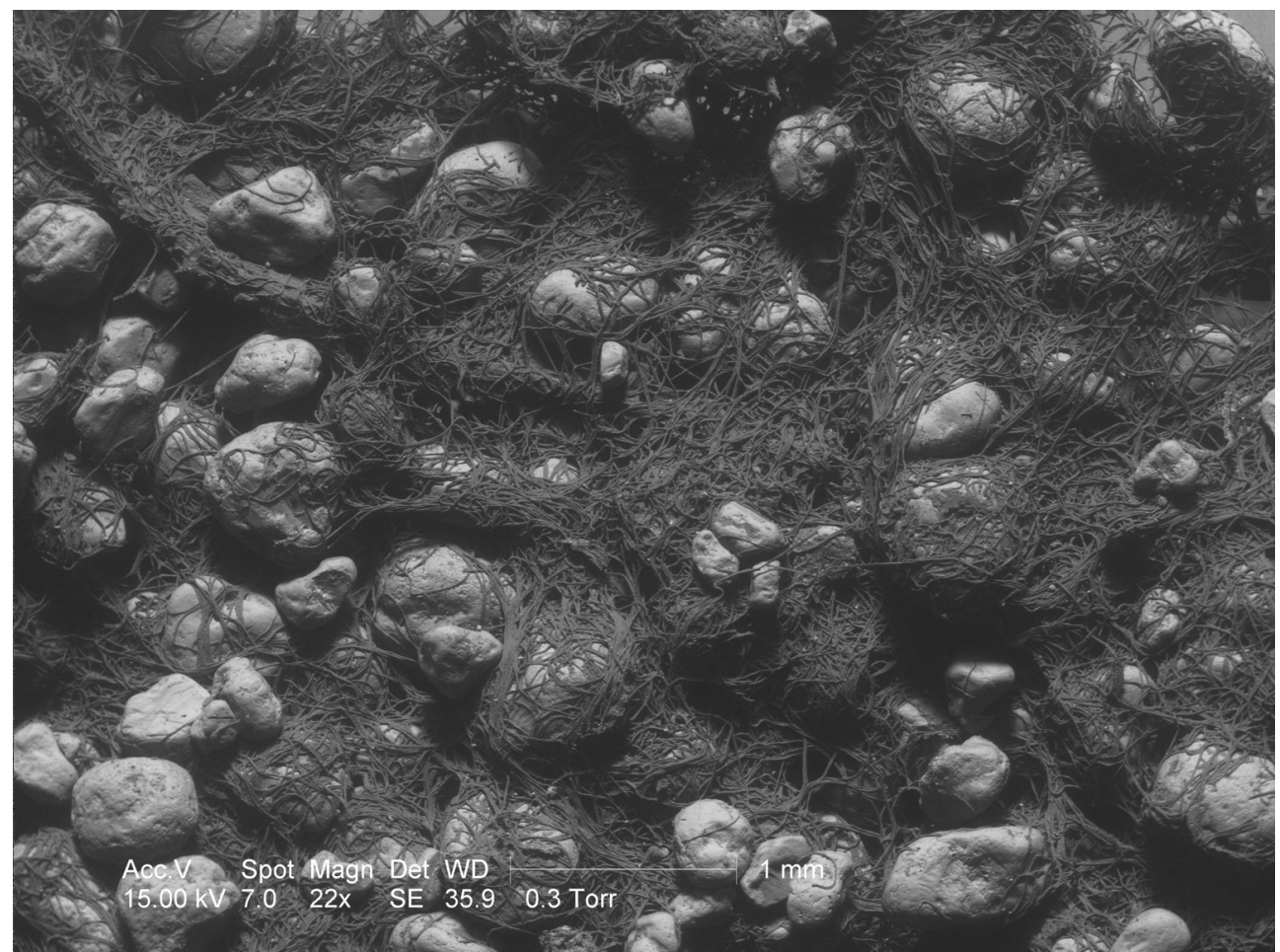

Fig. 2. Stabilization of loose sand by filamentous algae (BSE image)

In the soil-algal crust, 15 taxa were identified of which nine belong to Cyanophyta (Chroococcus minimus, Ch. minutus, Ch. varius, Gloeocapsa atrata, Merismopedia glauca, Synechococcus aeruginosus, Nostoc commune, Oscillatoria sp. and Phormidium sp.), one 
species belong to Heterokontophyta (Pinnularia borealis) and five to Chlorophyta (Stichococcus chlorelloides, S. cf. fragilis, Hormidiopsis crenulata, Cylindrocapsa sp. and Ulothrix sp.).

All species belonging to cyanobacteria, apart from Synechococcus aeruginosus, form small colonies surrounded by a gelatinous layer. Four of the species are unicellular forms (S. aeruginosus, $P$. borealis, $S$. chlorelloides, $S$. cf. fragilis), and four are filamentous forms (H. crenualtum, Cylindrocapsa sp., Osillatoria sp. and Phormidum sp.). They are common, forming characteristic woolly clusters of intertwined threads, ca. $0.0-1.0 \mathrm{~mm}$ thick, on the sand surface.

Greater morphological variability is shown by Chlorophyta occurring in unicellular, colonial and filamentous forms. Filamentous forms have the highest pedogenic significance due to their domination and their ability to stabilize loose sand by binding sand grains (Fig. 2).

\section{Physical and chemical properties of soil-algal crust}

Within analyzed samples the $0.4-0.25 \mathrm{~mm}$ sand fraction dominated in parent rock. The silt fraction is low, $1.3 \%$ on average; the only exception is sample number 2 with $18 \%$ (Table 1).

Table 1

The habitat features of soil-algal crusts

\begin{tabular}{|c|c|c|c|}
\hline $\begin{array}{l}\text { Sample } \\
\text { number }\end{array}$ & Description of sample sites & $\begin{array}{c}\text { Crust- } \\
\text { morphology type }\end{array}$ & $\begin{array}{l}\text { Thickness } \\
{[\mathrm{mm}]}\end{array}$ \\
\hline 1 & $\begin{array}{l}\text { southern slope of dune, crust covered by thin layer of } \\
\text { medium sand }(0.25-0.5 \mathrm{~mm}) \text {, green colour }\end{array}$ & \multirow{15}{*}{$\begin{array}{l}\text { All crusts consist } \\
\text { of species of soil } \\
\text { algae. In each case } \\
\text { crusts morphology } \\
\text { had flat type of } \\
\text { character }\end{array}$} & 1 \\
\hline 2 & $\begin{array}{l}\text { deflation field, fine sand }(0.10-0.25 \mathrm{~mm}) \text {, significant } \\
\text { fraction of silt }(0.002-0.05 \mathrm{~mm}) \text {, green }\end{array}$ & & 6 \\
\hline 3 & $\begin{array}{l}\text { deflation field, medium sand }(0.25-0.5 \mathrm{~mm}) \text {, green- and } \\
\text { blue-green algae are clearly visible in the humid period }\end{array}$ & & 1 \\
\hline 4 & $\begin{array}{l}\text { slightly hilly surface between young specimens of Pinus } \\
\text { sylvestris, medium sand }(0.25-0.5 \mathrm{~mm}) \text {, black and green } \\
\text { (coated by algae) }\end{array}$ & & 1 \\
\hline 5 & $\begin{array}{l}\text { northern slope of stabilized dune, medium sand } \\
(0.25-0.5 \mathrm{~mm}) \text {, black }\end{array}$ & & 4 \\
\hline 6 & phytogenic hillock, fine sand $(0.10-0.25 \mathrm{~mm})$, green & & 2 \\
\hline 7 & northern slope of dune, fine sand $(0.10-0.25 \mathrm{~mm})$, black & & 2 \\
\hline 8 & $\begin{array}{l}\text { flat stable sandy surface, medium sand } \\
(0.25-0.5 \mathrm{~mm}), \text { dark green }\end{array}$ & & 4 \\
\hline 9 & $\begin{array}{l}\text { northern slope of dune ridge, medium sand } \\
(0.25-0.5 \mathrm{~mm}) \text {, green }\end{array}$ & & 1 \\
\hline 10 & northern dune slope, fine sand, black color & & 4 \\
\hline 11 & loose- and medium sand $(0.25-0.5 \mathrm{~mm})$, dark green & & 3 \\
\hline 12 & loose- and medium sand $(0.25-0.5 \mathrm{~mm})$, dark green & & 2 \\
\hline 13 & loose- and medium sand $(0.25-0.5 \mathrm{~mm})$, dark green & & 3 \\
\hline 14 & $\begin{array}{l}\text { flat stable sandy surface, fine sand }(0.10-0.25 \mathrm{~mm}) \text {, dark } \\
\text { green }\end{array}$ & & 2 \\
\hline 15 & flat surface, medium sand $(0.25-0.5 \mathrm{~mm})$, black & & 4 \\
\hline
\end{tabular}

The organic carbon $\left(\mathrm{C}_{\mathrm{org}}\right)$ content in the soil-algal crusts varies from $0.35-2.23 \%$ (Table 2 ). The highest organic carbon content occurs in crusts enriched in clay (sample 2). The 
total nitrogen content $\left(\mathrm{N}_{\mathrm{t}}\right)$ increases with increasing $\mathrm{C}_{\text {org }}$. Available phosphorus $\left(\mathrm{P}_{\text {avail }}\right)$ also varies significantly in the range of $9-34 \mathrm{mg} \cdot \mathrm{kg}^{-1}$. Reaction of the investigated materials ranges from acidic ( $\mathrm{pH} 5.0-6.0$ in $\mathrm{H}_{2} \mathrm{O}$ ) to very acidic ( $\mathrm{pH} 4.4-5.7$ in $\mathrm{KCl}$; Table 2).

Table 2

Some physical and chemical properties of soil-algal crust $(n=3)$

\begin{tabular}{|c|c|c|c|c|c|c|}
\hline \multirow{2}{*}{$\begin{array}{l}\text { Sample } \\
\text { number }\end{array}$} & \multirow{2}{*}{$\begin{array}{c}\text { Thickness of } \\
\text { crust } \\
{[\mathrm{mm}]}\end{array}$} & $\mathrm{C}_{\text {org }}$ & $\mathbf{N}_{\mathrm{t}}$ & \multirow{2}{*}{$\begin{array}{c}P_{\text {avail }} \\
{\left[\mathrm{mg} \cdot \mathbf{k g}^{-1}\right]}\end{array}$} & \multicolumn{2}{|c|}{$\begin{array}{l}\mathbf{p H} \\
{[-]}\end{array}$} \\
\hline & & \multicolumn{2}{|c|}{$[\%]$} & & $\mathrm{KCl}$ & $\mathrm{H}_{2} \mathrm{O}$ \\
\hline 1 & 1 & 2.02 & 0.047 & 21 & 5.0 & 5.6 \\
\hline 2 & 6 & 2.23 & 0.113 & 20 & 4.3 & 5.2 \\
\hline 3 & 1 & 0.79 & 0.029 & 18 & 4.5 & 5.1 \\
\hline 4 & 1 & 1.91 & 0.130 & 13 & 4.4 & 5.3 \\
\hline 5 & 4 & 1.27 & 0.052 & 23 & 4.5 & 5.2 \\
\hline 6 & 2 & 2.12 & 0.049 & 34 & 4.5 & 5.3 \\
\hline 7 & 2 & 1.76 & 0.034 & 20 & 5.3 & 5.8 \\
\hline 8 & 4 & 1.50 & 0.066 & 34 & 4.4 & 5.1 \\
\hline 9 & 1 & 1.29 & 0.053 & 19 & 4.6 & 5.2 \\
\hline 10 & 4 & 0.80 & 0.041 & 24 & 5.4 & 6.0 \\
\hline 11 & 3 & 1.01 & 0.014 & 9 & 4.6 & 5.3 \\
\hline 12 & 2 & 0.64 & 0.019 & 37 & 5.7 & 6.1 \\
\hline 13 & 3 & 1.11 & 0.022 & 9 & 4.8 & 5.5 \\
\hline 14 & 2 & 1.25 & 0.017 & 12 & 4.4 & 5.0 \\
\hline 15 & 4 & 0.35 & 0.017 & 11 & 4.8 & 5.5 \\
\hline
\end{tabular}

Among mineral components in soil-algal crusts, iron dominates. Concentrations of iron vary greatly from place to place within the range of $776.5( \pm 2.3)-2803( \pm 31) \mathrm{mg} \cdot \mathrm{kg}^{-1}$ (Table $3)$. The next most important element present in the crusts is potassium with concentrations ranging from 230.5( \pm 3.7$)-696( \pm 22) \mathrm{mg} \cdot \mathrm{kg}^{-1}$. Concentrations of $\mathrm{Ca}$ and $\mathrm{Mg}$ are lower, from $47.1( \pm 9.3)-405( \pm 11) \mathrm{mg} \cdot \mathrm{kg}^{-1}$ and from $101.2( \pm 3.7)-314.4( \pm 1.4) \mathrm{mg} \cdot \mathrm{kg}^{-1}$, respectively. It is worth noting that the soil-algal crusts are characterized by very low sodium ocntent, often near to the detection limit (Table 3) and even the highest sodium concentrations $\left(98.8( \pm 6.7) \mathrm{mg} \cdot \mathrm{kg}^{-1}\right)$ are relatively low. However, zinc and manganese concentrations are high at $16.7( \pm 1.1)-118( \pm 12) \mathrm{mg} \cdot \mathrm{kg}^{-1}$ and $9.70( \pm 1.03)-47.69( \pm 0.17) \mathrm{mg} \cdot \mathrm{kg}^{-1}$, respectively.

As lead concentrations in most samples $\left(10.50( \pm 0.30)+222.1( \pm 1.2) \mathrm{mg} \cdot \mathrm{kg}^{-1}\right)$ exceed geochemical background values $\left(13 \mathrm{mg} \cdot \mathrm{kg}^{-1}\right)$, there is a possibility that heavy metals may accumulate at the early stage of soil formation due to the occurrence of soil-algal crust. Cadmium concentrations are low and occur in the range of of $0.102( \pm 0.001)-0.776( \pm 0.011) \mathrm{mg} \cdot \mathrm{kg}^{-1}$ (Table 3).

Table 3

Average concentration $(n=5)$ of elements in soil-algal crust from Bledowska Desert

\begin{tabular}{|c|c|c|c|c|c|c|c|c|c|c|c|}
\hline $\begin{array}{c}\text { Sample } \\
\text { number }\end{array}$ & $\mathbf{C a}$ & $\mathbf{M g}$ & $\mathbf{K}$ & $\mathbf{N a}$ & $\mathbf{C u}$ & $\mathbf{Z n}$ & $\mathbf{P b}$ & $\mathbf{M n}$ & $\mathbf{F e}$ & $\mathbf{C d}$ \\
\hline & \multicolumn{10}{|c|}{$\left[\mathbf{m g} \cdot \mathbf{k g}^{-1}\right]$} \\
\hline 1 & $\begin{array}{c}207.0 \\
( \pm 8.1)^{\mathrm{efg}}\end{array}$ & $\begin{array}{c}220.1 \\
( \pm 3.9)^{\mathrm{abc}}\end{array}$ & $\begin{array}{c}696 \\
( \pm 22)^{\mathrm{d}}\end{array}$ & $\begin{array}{c}36.0 \\
( \pm 4.3)^{\mathrm{d}}\end{array}$ & $\begin{array}{c}4.77 \\
( \pm 0.06)^{\mathrm{f}}\end{array}$ & $\begin{array}{c}101.24 \\
( \pm 0.10)^{\mathrm{g}}\end{array}$ & $\begin{array}{c}111.9 \\
( \pm 2.1)^{\mathrm{j}}\end{array}$ & $\begin{array}{c}18.25 \\
( \pm 0.23)^{\mathrm{c}}\end{array}$ & $\begin{array}{c}2029 \\
( \pm 126)^{\mathrm{cde}}\end{array}$ & $\begin{array}{c}0.568 \\
( \pm 0.031)^{\mathrm{h}}\end{array}$ \\
\hline \multirow{2}{*}{2} & $\begin{array}{c}121.7 \\
( \pm 2.1)^{\mathrm{cd}}\end{array}$ & $\begin{array}{c}136.2 \\
( \pm 3.9)^{\mathrm{ab}}\end{array}$ & $\begin{array}{c}357.8 \\
( \pm 3.4)^{\mathrm{ab}}\end{array}$ & $\left\langle\mathrm{LOQ}^{* *}\right.$ & $\begin{array}{c}1.61 \\
( \pm 0.54)^{\mathrm{a}}\end{array}$ & $\begin{array}{c}16.7 \\
( \pm 1.1)^{\mathrm{a}}\end{array}$ & $\begin{array}{c}10.50 \\
( \pm 0.30)^{\mathrm{a}}\end{array}$ & $\begin{array}{c}10.99 \\
( \pm 0.13)^{\mathrm{ab}}\end{array}$ & $\begin{array}{c}886 \\
( \pm 29)^{\mathrm{a}}\end{array}$ & $\begin{array}{c}0.106 \\
( \pm 0.004)^{\mathrm{a}}\end{array}$ \\
\hline
\end{tabular}




\begin{tabular}{|c|c|c|c|c|c|c|c|c|c|c|}
\hline \multirow{2}{*}{$\begin{array}{l}\text { Sample } \\
\text { number }\end{array}$} & Ca & Mg & $\mathbf{K}$ & $\mathbf{N a}$ & \begin{tabular}{l|l}
$\mathbf{C u}$ \\
\end{tabular} & $\mathbf{Z n}$ & $\mathbf{P b}$ & Mn & $\mathbf{F e}$ & Cd \\
\hline & \multicolumn{10}{|c|}{$\left[\mathrm{mg} \cdot \mathrm{kg}^{-1}\right]$} \\
\hline 3 & $\begin{array}{c}250 \\
( \pm 20)^{\mathrm{g}}\end{array}$ & $\begin{array}{c}246.4 \\
( \pm 9.6)^{\mathrm{abc}}\end{array}$ & $\begin{array}{c}626 \\
( \pm 25)^{\mathrm{cd}}\end{array}$ & $\begin{array}{c}30.4 \\
( \pm 1.9)^{\mathrm{cd}}\end{array}$ & $\begin{array}{c}5.61 \\
( \pm 0.12)^{\mathrm{f}}\end{array}$ & $\begin{array}{c}117.1 \\
( \pm 2.5)^{\mathrm{h}}\end{array}$ & $\begin{array}{c}222.1 \\
( \pm 1.2)^{\mathrm{m}}\end{array}$ & $\begin{array}{c}28.46 \\
( \pm 0.72)^{d}\end{array}$ & $\begin{array}{c}2803 \\
( \pm 31)^{g}\end{array}$ & $\begin{array}{c}0.776 \\
( \pm 0.011)^{\mathrm{i}}\end{array}$ \\
\hline 4 & $\begin{array}{c}192.3 \\
( \pm 9.4)^{\mathrm{efg}}\end{array}$ & $\begin{array}{c}101.2 \\
( \pm 3.7)^{\mathrm{a}}\end{array}$ & $\begin{array}{c}283.9 \\
( \pm 7.7)^{\mathrm{ab}}\end{array}$ & $\begin{array}{c}12.81 \\
( \pm 0.54)^{b}\end{array}$ & $\begin{array}{c}2.09 \\
( \pm 0.21)^{\mathrm{abc}}\end{array}$ & $\begin{array}{c}28.0 \\
( \pm 1.8)^{b}\end{array}$ & $\begin{array}{c}39.97 \\
( \pm 0.48)^{\mathrm{e}}\end{array}$ & $\begin{array}{c}11.24 \\
( \pm 0.46)^{\mathrm{ab}}\end{array}$ & $\begin{array}{c}867 \\
( \pm 25)^{\mathrm{a}}\end{array}$ & $\begin{array}{c}0.153 \\
( \pm 0.011)^{b}\end{array}$ \\
\hline 5 & $\begin{array}{c}199 \\
( \pm 15)^{\mathrm{efg}}\end{array}$ & $\begin{array}{c}205.9 \\
( \pm 8.5)^{\mathrm{abc}}\end{array}$ & $\begin{array}{c}664.26 \\
( \pm 0.48)^{d}\end{array}$ & $\begin{array}{c}98.8 \\
( \pm 6.7)^{\mathrm{f}}\end{array}$ & $\begin{array}{c}2.98 \\
( \pm 0.20)^{\mathrm{bcd}} \\
\mathrm{e}\end{array}$ & $\begin{array}{c}118 \\
( \pm 12)^{\mathrm{h}}\end{array}$ & $\begin{array}{c}185.3 \\
( \pm 6.5)^{1}\end{array}$ & $\begin{array}{c}19.96 \\
( \pm 0.19)^{\mathrm{c}}\end{array}$ & $\begin{array}{c}1822 \\
( \pm 61)^{b c d}\end{array}$ & $\begin{array}{c}0.589 \\
( \pm 0.005)^{h}\end{array}$ \\
\hline 6 & $\begin{array}{c}171 \\
( \pm 16)^{\mathrm{def}}\end{array}$ & $\begin{array}{c}275.8 \\
( \pm 4.1)^{b} \\
\end{array}$ & $\begin{array}{c}357.8 \\
( \pm 7.8)^{\mathrm{ab}}\end{array}$ & $<\mathrm{LOQ}^{* * *}$ & $\begin{array}{c}3.05 \\
( \pm 0.32)^{\mathrm{cde}}\end{array}$ & $\begin{array}{c}75.1 \\
( \pm 2.7)^{\mathrm{e}}\end{array}$ & $\begin{array}{c}56.8 \\
( \pm 1.3)^{\mathrm{g}} \\
\end{array}$ & $\begin{array}{c}47.69 \\
( \pm 0.17)^{\mathrm{g}} \\
\end{array}$ & $\begin{array}{c}2180 \\
( \pm 61)^{\text {def }}\end{array}$ & $\begin{array}{c}0.473 \\
( \pm 0.011)^{\mathrm{g}}\end{array}$ \\
\hline 7 & $\begin{array}{c}236 \\
( \pm 17)^{\mathrm{fg}}\end{array}$ & $\begin{array}{c}165.0 \\
( \pm 1.2)^{\mathrm{abc}}\end{array}$ & $\begin{array}{c}529 \\
( \pm 14)^{\mathrm{bcd}}\end{array}$ & $\begin{array}{c}68.1 \\
( \pm 8.0)^{\mathrm{e}}\end{array}$ & $\begin{array}{c}3.59 \\
( \pm 0.72)^{\mathrm{e}}\end{array}$ & $\begin{array}{c}83.3 \\
( \pm 1.2)^{\mathrm{f}}\end{array}$ & $\begin{array}{c}129.3 \\
( \pm 2.7)^{\mathrm{k}}\end{array}$ & $\begin{array}{c}16.36 \\
( \pm 0.34)^{\mathrm{bc}}\end{array}$ & $\begin{array}{l}1473.2 \\
( \pm 5.0)^{b}\end{array}$ & $\begin{array}{c}0.322 \\
( \pm 0.013)^{\mathrm{e}}\end{array}$ \\
\hline 8 & $\begin{array}{c}165.9 \\
( \pm 5.5)^{\mathrm{de}}\end{array}$ & $\begin{array}{c}259.8 \\
( \pm 4.9)^{\mathrm{b}}\end{array}$ & $\begin{array}{c}396 \\
( \pm 14)^{\mathrm{abc}}\end{array}$ & $<\mathrm{LOQ}^{* *}$ & $\begin{array}{c}3.32 \\
( \pm 0.15)^{\mathrm{e}}\end{array}$ & $\begin{array}{c}80.4 \\
( \pm 1.1)^{\text {ef }}\end{array}$ & $\begin{array}{c}67.3 \\
( \pm 1.5)^{\mathrm{h}} \\
\end{array}$ & $\begin{array}{c}36.06 \\
( \pm 0.98)^{\mathrm{f}}\end{array}$ & $\begin{array}{l}2287 \\
( \pm 19)^{\text {ef }}\end{array}$ & $\begin{array}{c}0.342 \\
( \pm 0.007)^{\mathrm{e}}\end{array}$ \\
\hline 9 & $\begin{array}{c}98.93 \\
( \pm 0.86)^{\mathrm{bc}}\end{array}$ & $\begin{array}{c}246 \\
( \pm 10)^{\mathrm{abc}}\end{array}$ & $\begin{array}{c}443.5 \\
( \pm 8.1)^{\text {abcd }}\end{array}$ & $<\mathrm{LOQ}^{* *}$ & $\begin{array}{c}2.90 \\
( \pm 0.50)^{\mathrm{abc}} \\
\mathrm{de}\end{array}$ & $\begin{array}{c}60.99 \\
( \pm 0.86)^{\mathrm{d}}\end{array}$ & $\begin{array}{c}48.57 \\
( \pm 0.86)^{\mathrm{f}}\end{array}$ & $\begin{array}{c}29.84 \\
( \pm 0.49)^{\mathrm{de}}\end{array}$ & $\begin{array}{c}1709 \\
( \pm 124)^{b c}\end{array}$ & $\begin{array}{c}0.349 \\
( \pm 0.030)^{\mathrm{e}}\end{array}$ \\
\hline 10 & $\begin{array}{c}328.15 \\
( \pm 0.25)^{\mathrm{h}}\end{array}$ & $\begin{array}{c}152.5 \\
( \pm 1.7)^{\mathrm{ab}}\end{array}$ & $\begin{array}{c}252.46 \\
( \pm 0.54)^{\mathrm{a}}\end{array}$ & $<\mathrm{LOQ}^{* * *}$ & $\begin{array}{c}1.99 \\
( \pm 0.58)^{\mathrm{ab}}\end{array}$ & $\begin{array}{c}28.4 \\
( \pm 1.0)^{\mathrm{b}}\end{array}$ & $\begin{array}{c}19.50 \\
( \pm 0.15)^{\mathrm{cd}}\end{array}$ & $\begin{array}{c}11.66 \\
( \pm 0.81)^{\mathrm{ab}}\end{array}$ & $\begin{array}{c}946 \\
( \pm 20)^{\mathrm{a}}\end{array}$ & $\begin{array}{c}0.164 \\
( \pm 0.001)^{\mathrm{c}}\end{array}$ \\
\hline 11 & $\begin{array}{c}405 \\
( \pm 11)^{\mathrm{i}} \\
\end{array}$ & $\begin{array}{c}267.9 \\
( \pm 3.6)^{b}\end{array}$ & $\begin{array}{c}402.7 \\
( \pm 4.7)\end{array}$ & $<\mathrm{LOQ}^{* * *}$ & $\begin{array}{c}2.12 \\
( \pm 0.62)^{\mathrm{abc}}\end{array}$ & $\begin{array}{c}55.14 \\
( \pm 0.04)^{d}\end{array}$ & $\begin{array}{c}40.17 \\
( \pm 0.11)^{\mathrm{e}}\end{array}$ & $\begin{array}{c}27.03 \\
( \pm 0.54)^{d}\end{array}$ & $\begin{array}{c}1507 \\
( \pm 48)^{b}\end{array}$ & $\begin{array}{c}0.288 \\
( \pm 0.008)^{d}\end{array}$ \\
\hline 12 & $\begin{array}{c}65 \\
( \pm 12)^{\mathrm{bc}}\end{array}$ & $\begin{array}{c}208.3 \\
( \pm 6.6)^{\mathrm{abc}}\end{array}$ & $\begin{array}{c}330 \\
( \pm 12)^{\mathrm{ab}}\end{array}$ & $<\mathrm{LOQ}^{* *}$ & $\begin{array}{c}3.22 \\
( \pm 0.17)^{\mathrm{de}}\end{array}$ & $\begin{array}{c}41.6 \\
( \pm 2.9)^{\mathrm{c}} \\
\end{array}$ & $\begin{array}{c}23.96 \\
( \pm 0.68)^{d}\end{array}$ & $\begin{array}{c}36.9 \\
( \pm 2.7)^{\mathrm{f}} \\
\end{array}$ & $\begin{array}{c}1623 \\
( \pm 41)^{\mathrm{bc}}\end{array}$ & $\begin{array}{c}0.282 \\
( \pm 0.007)^{d}\end{array}$ \\
\hline 13 & $<\mathrm{LOQ}^{*}$ & $\begin{array}{c}133.7 \\
( \pm 5.5)^{\mathrm{ab}}\end{array}$ & $\begin{array}{l}230.5 \\
( \pm 3.7)^{\mathrm{a}}\end{array}$ & $\begin{array}{c}24.2 \\
( \pm 3.5)^{\mathrm{c}}\end{array}$ & $\begin{array}{c}1.94 \\
( \pm 0.18)^{\mathrm{a}}\end{array}$ & $\begin{array}{c}24.06 \\
( \pm 0.32)^{\mathrm{ab}}\end{array}$ & $\begin{array}{c}15.65 \\
( \pm 0.54)^{\mathrm{bc}}\end{array}$ & $\begin{array}{c}9.83 \\
( \pm 0.31)^{\mathrm{a}}\end{array}$ & $\begin{array}{l}776.5 \\
( \pm 2.3)^{\mathrm{a}}\end{array}$ & $\begin{array}{c}0.102 \\
( \pm 0.001)^{\mathrm{a}}\end{array}$ \\
\hline 14 & $\begin{array}{c}47.1 \\
( \pm 9.3)^{\mathrm{ab}}\end{array}$ & $\begin{array}{c}162.7 \\
( \pm 3.3)^{\mathrm{abc}}\end{array}$ & $\begin{array}{c}277.3 \\
( \pm 17.1)^{\mathrm{ab}}\end{array}$ & $<\mathrm{LOQ}^{* *}$ & $\begin{array}{c}2.29 \\
( \pm 0.16)^{\mathrm{abc}} \\
\mathrm{d}\end{array}$ & $\begin{array}{c}20.75 \\
( \pm 0.09)^{\mathrm{ab}}\end{array}$ & $\begin{array}{c}11.94 \\
( \pm 0.06)^{\mathrm{ab}}\end{array}$ & $\begin{array}{c}9.70 \\
( \pm 1.03)^{\mathrm{a}}\end{array}$ & $\begin{array}{c}856.09 \\
( \pm 0.69)^{\mathrm{a}}\end{array}$ & $\begin{array}{c}0.127 \\
( \pm 0.001)^{\mathrm{a}} \\
\mathrm{b}\end{array}$ \\
\hline 15 & $\begin{array}{c}208.7 \\
( \pm 3.5)^{\text {efg }}\end{array}$ & $\begin{array}{c}314.4 \\
( \pm 1.4)^{\mathrm{cd}}\end{array}$ & \begin{tabular}{|c}
476.3 \\
$( \pm 3.8)^{\mathrm{abcd}}$
\end{tabular} & $\begin{array}{c}10.6 \\
( \pm 4.3)^{b}\end{array}$ & $\begin{array}{c}4.71 \\
( \pm 0.07)^{\mathrm{f}}\end{array}$ & $\begin{array}{c}78.7 \\
( \pm 1.3)^{\mathrm{ef}}\end{array}$ & $\begin{array}{c}72.9 \\
( \pm 4.2)^{\mathrm{i}}\end{array}$ & $\begin{array}{c}34.90 \\
( \pm 0.09)^{\mathrm{ef}}\end{array}$ & $\begin{array}{c}2499 \\
( \pm 15)^{\mathrm{fg}}\end{array}$ & $\begin{array}{c}0.395 \\
( \pm 0.003)^{f}\end{array}$ \\
\hline
\end{tabular}

The same letter $(a, b, c, d, e, f, g, h, i, j, k, 1, m)$ in superscript means no significant differences according to the Duncan test $(p<0.05)$

* limit of quantification of $\mathrm{Ca}=5.02 \mathrm{mg} / \mathrm{kg}$

** limit of quantification of $\mathrm{Na}=1.36 \mathrm{mg} / \mathrm{kg}$

\section{Curie-point Py-GC/MS data}

Pyrolityc analyses of soil-algal crusts showed the occurrences of following organic compound belonging to different groups ie cyclic and acyclic aliphatic hydrocarbons, aromatic hydrocarbons, carbohydrate derivatives, organic compounds containing nitrogen and sulphur, phenols, prenyl, furan and pyrane derivatives (Table 4).

Table 4

List of main pyrolysis products of soil-algal-crust

\begin{tabular}{|c|c|c|c|c|}
\hline $\begin{array}{c}\text { Area } \\
{[\%]}\end{array}$ & Compound derived from pyrolysis product & Source & $\mathbf{M}^{+}$ & Formula \\
\hline 0.95 & 2-Methyl-1-propene & $\mathrm{Ps}$ & 56 & $\mathrm{C}_{4} \mathrm{H}_{8}$ \\
\hline 1.64 & Butane & $\mathrm{Ps}$ & 58 & $\mathrm{C}_{4} \mathrm{H}_{10}$ \\
\hline 0.84 & 1-Propanol & $\mathrm{Ps}$ & 60 & $\mathrm{C}_{3} \mathrm{H}_{8} \mathrm{O}$ \\
\hline 0.74 & 2,3-Dihydro-furan & $\mathrm{Ps}$ & 70 & $\mathrm{C}_{4} \mathrm{H}_{6} \mathrm{O}$ \\
\hline 0.58 & Pyrrolidine & $\mathrm{Pr}$ & 71 & $\mathrm{C}_{4} \mathrm{H}_{9} \mathrm{~N}$ \\
\hline 0.89 & Oxiranemethanol & $\mathrm{Ps} / \mathrm{Lg}$ & 74 & $\mathrm{C}_{3} \mathrm{H}_{6} \mathrm{O}_{2}$ \\
\hline
\end{tabular}




\begin{tabular}{|c|c|c|c|c|}
\hline $\begin{array}{c}\text { Area } \\
{[\%]}\end{array}$ & Compound derived from pyrolysis product & Source & $\mathbf{M}^{+}$ & Formula \\
\hline 2.63 & 2-Amino-1-propanol & $\mathrm{Ps} / \mathrm{Pr}$ & 75 & $\mathrm{C}_{3} \mathrm{H}_{9} \mathrm{NO}$ \\
\hline 0.18 & Benzene & $\mathrm{Lg}$ & 78 & $\mathrm{C}_{6} \mathrm{H}_{6}$ \\
\hline 1.5 & 1-Hexene & Ps & 84 & $\mathrm{C}_{6} \mathrm{H}_{12}$ \\
\hline 1.27 & Nitrosoazetidine & $\operatorname{Pr}$ & 86 & $\mathrm{C}_{3} \mathrm{H}_{6} \mathrm{~N}_{2} \mathrm{O}$ \\
\hline 0.57 & 2-Amino-1-butanol & $\mathrm{Ps} / \mathrm{Pr}$ & 89 & $\mathrm{C}_{4} \mathrm{H}_{11} \mathrm{NO}$ \\
\hline 0.61 & Toluene & $\mathrm{Lg}$ & 92 & $\mathrm{C}_{7} \mathrm{H}_{8}$ \\
\hline 0.74 & 2H-Pyran-2-one & Ps & 96 & $\mathrm{C}_{5} \mathrm{H}_{4} \mathrm{O}_{2}$ \\
\hline 0.68 & 2-Furanmethanol & Ps & 98 & $\mathrm{C}_{5} \mathrm{H}_{6} \mathrm{O}_{2}$ \\
\hline 0.52 & 3,5-Dimethyl-pyrazol & $\operatorname{Pr}$ & 96 & $\mathrm{C}_{5} \mathrm{H}_{8} \mathrm{~N}_{2}$ \\
\hline 1.07 & 2 Methyl pentanal & Ps & 100 & $\mathrm{C}_{6} \mathrm{H}_{12} \mathrm{O}$ \\
\hline 0.51 & N-ethyl-N-nitroso-ethanamine & $\operatorname{Pr}$ & 102 & $\mathrm{C}_{4} \mathrm{H}_{10} \mathrm{~N}_{2} \mathrm{O}$ \\
\hline 0.16 & Ethylbenzene & $\mathrm{Lg}$ & 106 & $\mathrm{C}_{8} \mathrm{H}_{10}$ \\
\hline 3.00 & 2-Heptanone & $\mathrm{Ps} / \mathrm{p}$ & 114 & $\mathrm{C}_{7} \mathrm{H}_{14} \mathrm{O}$ \\
\hline 1.39 & N,N'-Dimethylpiperazine & $\operatorname{Pr}$ & 114 & $\mathrm{C}_{6} \mathrm{H}_{14} \mathrm{~N}_{2}$ \\
\hline 0.80 & Acryloylurea & $\operatorname{Pr}$ & 114 & $\mathrm{C}_{4} \mathrm{H}_{6} \mathrm{~N}_{2} \mathrm{O}_{2}$ \\
\hline 0.86 & 2-Methyliminoperhydro-1,3-oxazine & $\mathrm{Pr} / \mathrm{s}$ & 114 & $\mathrm{C}_{5} \mathrm{H}_{10} \mathrm{~N}_{2} \mathrm{O}$ \\
\hline 5.02 & 1,2-Dihydro-4-methyl-3,6-pyridazinedione & $\mathrm{Ps} / \mathrm{Pr}$ & 126 & $\mathrm{C}_{5} \mathrm{H}_{6} \mathrm{~N}_{2} \mathrm{O}_{2}$ \\
\hline 1.67 & 3,4-Anhydro-d-galactosan & Ps & 144 & $\mathrm{C}_{6} \mathrm{H}_{8} \mathrm{O}_{4}$ \\
\hline 0.44 & N-Nitroso-2,4,4-trimethyloxazolidine & $\operatorname{Pr}$ & 144 & $\mathrm{C}_{6} \mathrm{H}_{12} \mathrm{~N}_{2} \mathrm{O}_{2}$ \\
\hline 0.91 & Dihydroxymaleic acid & $\mathrm{Ps} / \mathrm{Lg}$ & 148 & $\mathrm{C}_{4} \mathrm{H}_{4} \mathrm{O}_{6}$ \\
\hline 0.72 & N,N-Diethyl-N'-propylacetamdine & $\operatorname{Pr}$ & 156 & $\mathrm{C}_{9} \mathrm{H}_{20} \mathrm{~N}_{2}$ \\
\hline 13.71 & 1,6-Anhydro- $\beta$-D-glucopyranose (levoglucosan) & Ps & 162 & $\mathrm{C}_{6} \mathrm{H}_{10} \mathrm{O}_{5}$ \\
\hline 2.81 & 3-Methylmannoside & Ps & 194 & $\mathrm{C}_{7} \mathrm{H}_{14} \mathrm{O}_{6}$ \\
\hline 0.38 & Pentadecane & Lp & 212 & $\mathrm{C}_{15} \mathrm{H}_{32}$ \\
\hline 10.15 & 1-Gala-1-ido-octonic lactone & Ps/Lp & 238 & $\mathrm{C}_{8} \mathrm{H}_{14} \mathrm{O}_{8}$ \\
\hline 3.81 & 8-Heptadecene & $\mathrm{Lp}$ & 238 & $\mathrm{C}_{17} \mathrm{H}_{34}$ \\
\hline 1.93 & Cytidine & $\operatorname{Pr}$ & 243 & $\mathrm{C}_{9} \mathrm{H}_{13} \mathrm{~N}_{3} \mathrm{O}_{5}$ \\
\hline 0.66 & Hexadecanoic acid & Lp & 256 & $\mathrm{C}_{16} \mathrm{H}_{32} \mathrm{O}_{2}$ \\
\hline 17.4 & Ethyl(-2-butylthio) ester of 2-pentyl-cyclopropanoic acid & PS/Lp & 272 & $\mathrm{C}_{15} \mathrm{H}_{28} \mathrm{O}_{2} \mathrm{~S}$ \\
\hline 0.20 & $\begin{array}{l}\text { (E)- 5-Eicosene } \\
\end{array}$ & Lp & 280 & $\mathrm{C}_{20} \mathrm{H}_{40}$ \\
\hline 0.46 & Oleic acid & Lp & 282 & $\mathrm{C}_{18} \mathrm{H}_{34} \mathrm{O}_{2}$ \\
\hline 0.84 & 2-Vinyl-9-[ $\beta$-d-ribofuranosyl] hypoxanthine & $\mathrm{Ps} / \mathrm{Lg}$ & 294 & $\mathrm{C}_{12} \mathrm{H}_{14} \mathrm{~N}_{4} \mathrm{O}_{5}$ \\
\hline 0.89 & 2-Formyl-9-[ $\beta$-d-ribofuranosyl] hypoxanthine & $\mathrm{Ps} / \mathrm{Pr}$ & 296 & $\mathrm{C}_{11} \mathrm{H}_{12} \mathrm{~N}_{4} \mathrm{O}_{6}$ \\
\hline 1.17 & 2-Hydroxymethyl-9-[ $\beta$-d-ribofuranosyl] hypoxanthine & $\mathrm{Ps} / \mathrm{Pr}$ & 298 & $\mathrm{C}_{11} \mathrm{H}_{14} \mathrm{~N}_{4} \mathrm{O}_{6}$ \\
\hline 0.66 & Isopropyl Palmitate & Lp & 298 & $\mathrm{C}_{19} \mathrm{H}_{38} \mathrm{O}_{2}$ \\
\hline 0.20 & Docosanoic acid & Lp & 340 & $\mathrm{C}_{22} \mathrm{H}_{44} \mathrm{O}_{2}$ \\
\hline
\end{tabular}

Aliphatic compounds, both cyclic and acyclic, include saturated- and unsaturated hydrocarbons, alcohols and carboxylic acids from various sources such as polysaccharides and lipids. Organic compounds with aromatic rings, such as toluene and benzene, are present in low concentrations in analyzed soil-algal crust material. Furan and pyrane derivatives occur only rarely in trace amounts in samples. Among organic components, the highest concentrations are shown by polysaccharides source of which the most important are compounds identified as 1,6-anhydro- $\beta$-D-glucopyranose (levoglucosan), 3-methylmannoside, 3,4-anhydro-d-galactosane and others - all estimated from peaks areas (Table 4).

\section{Microscopic investigations (SEM)}

During the initial stages of soil-algal crust formation, characteristic elongated forms (often from filamentous algae) are created with rare mineral grains or aggregates which are 
attached to them (Fig. 3a, c, d). In the investigated soil-algal crusts, metal-bearing mineral grains are mainly iron oxides (Fig. 3a, c).

a)

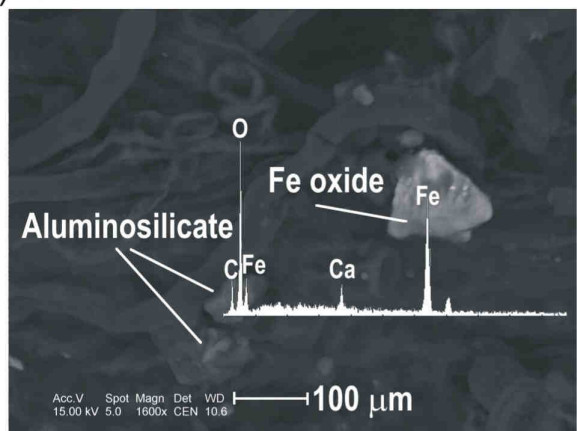

c)

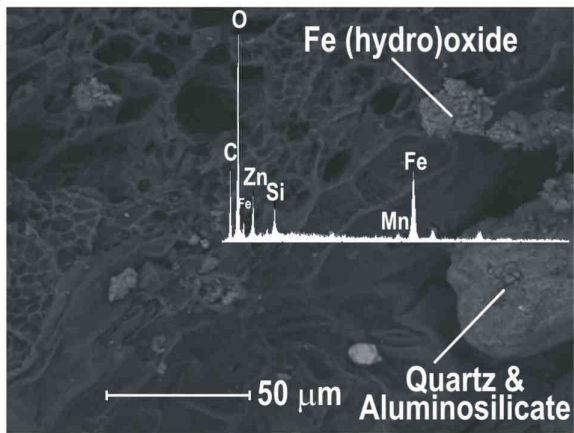

b)

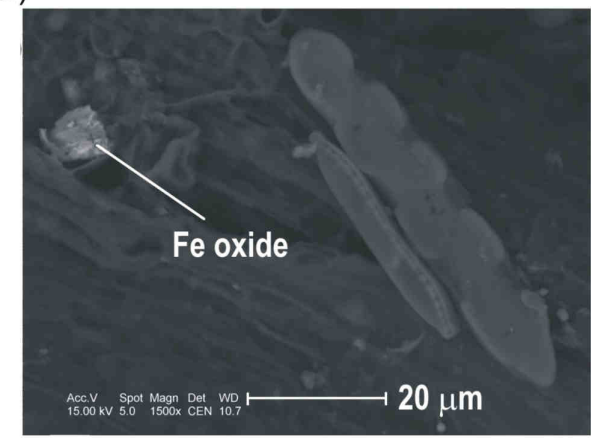

d)

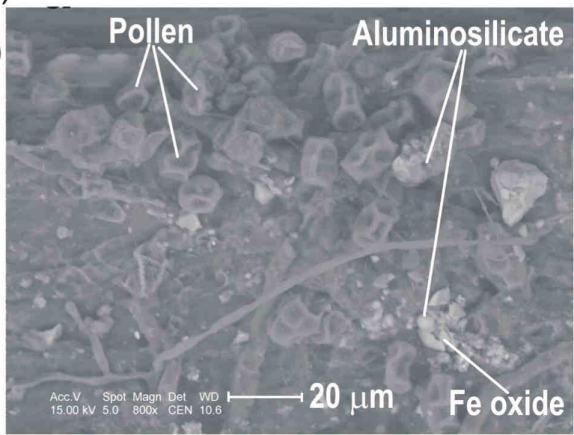

Fig. 3. BSE images of algal-soil crust: a) algal matter with Fe oxide grains identified by EDS analysis; b) algal matter and $\mathrm{Fe}$ oxide grain; c) algal matter and $\mathrm{Fe}$ (hydro)oxide grain $\mathrm{Zn}$ and Mn-bearing, identified by EDS analysis; d) algal matter, pollen and Fe oxide

Well-developed soil-algal crusts have complex structure and diverse chemical composition. Apart from algal filaments, there are other biogenic components present, $e g$ fungi hyphae, spores and pollens, roots of plants and aggregates of microorganisms. All of the algal-soil crusts investigated contain both organic matter represented by algae fragments, fungi hyphae, roots, pollens, microorganisms and their excreta (Fig. 3d) and allochtonic minerals trapped in a complex algal net - the most important crustal component. In the crusts, the most abundant minerals are quartz and aluminosilicates occurring as fine-grained clay-mineral aggregates, quartz grains and feldspars. Mineral grains and aggregates containing metals can occur as well. Large, relatively common, aggregates of iron oxides are often associated with aluminosilicates (Fig. 3a, b, c, d). Soil-algal crusts forming thicker coverings contain higher proportions of inorganic mineral components (Fig. 4a, d). At more advanced stages of their development, organo-mineral crusts contain minerals (aluminosilicates, quartz, Fe oxides) in considerable quantities. In some cases, it is difficult to recognize algae in mineral crusts bound by aluminosilicates and in thick nets 
comprising fragments of algae, fungi and roots. In the formation of such crusts, an important role is played by organic excreta and polymeric nets of algae-fungi.

In BSE images of the organo-mineral crusts, there are pale mineral grains rich in $\mathrm{Fe}$, $\mathrm{Zn}, \mathrm{Mg}, \mathrm{Ca}$ and $\mathrm{K}$ (Fig. 4b, c, d). In soil-algal crusts formed at the eastern edge of the desert, close to the emission sources related to smelting and mining, there are also metal-bearing phases rich in $\mathrm{Zn}, \mathrm{Pb}$ and $\mathrm{Mn}$. Their identification is possible at high magnification $(>1000 \mathrm{x})$. The presence of other metals was confirmed by AAS (Table 3 ).

a)

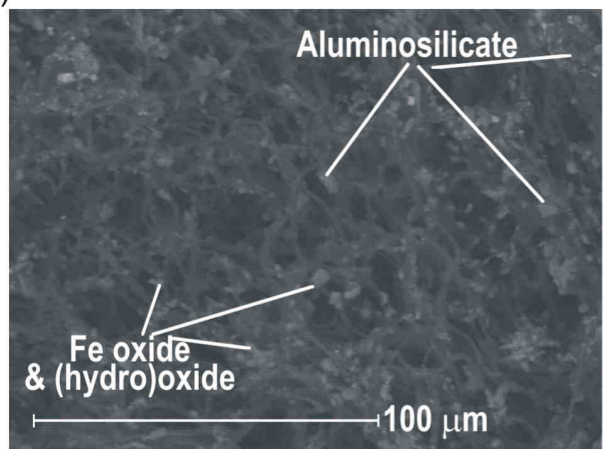

C)

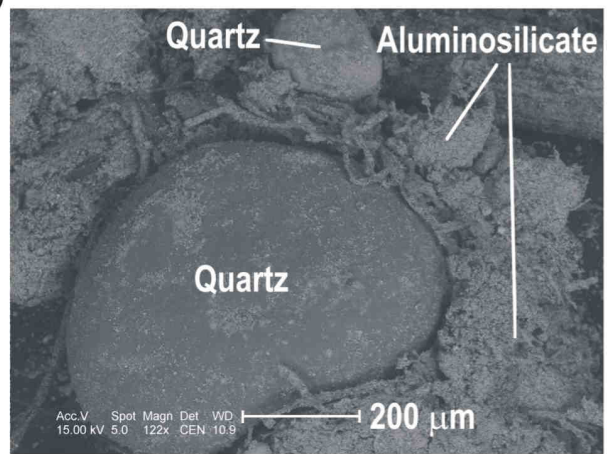

b)

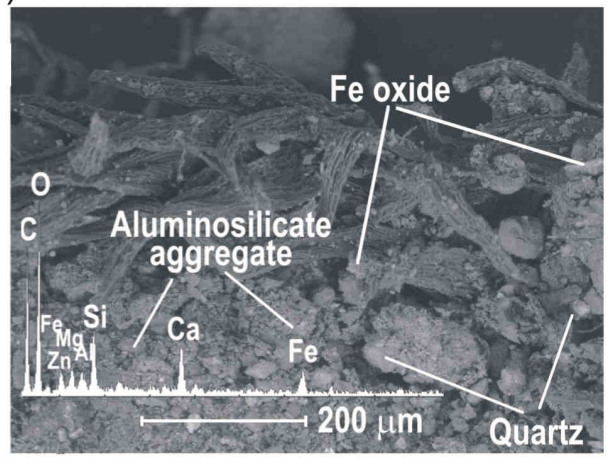

d)

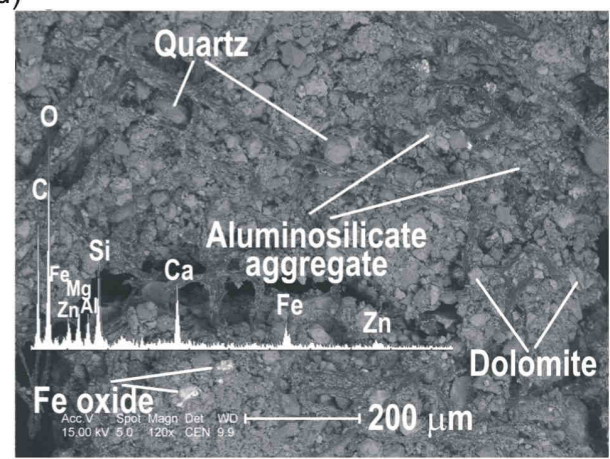

Fig. 4. BSE images of algal-soil crust: a) netlike algal matter with Fe oxide and (hydro)oxide grains, b) algal matter on poly-mineral ground with metal-bearing aggregates identified by EDS analysis, c) quartz grains surrounded by aluminosilicates and algal matter, d) mineral and algal matter with metal-bearing grains identified by EDS analysis

In the initial stage of soil-algal crusts development in the net of filamentous a few grains of Fe oxide occur (Fig. 3a, b). The forming of algal net increase the trapping rate of fine mineral grains (Fig. 4a). A fully developed soil-algal crust is characterized by the presence of compacted aluminosilicate, carbonate and metalliferous mineral aggregates which are sticking to different biotic components (Fig. 4b, c, d). 


\section{Discussion}

Drift sand or stabilized sandy areas with low contents of fine-grained fractions do not provide good conditions for living organisms due to low nutrient concentrations, low water retention and low sorption capacity. On a mineral substrate, microscopic cyanobacteria (Chroococcus minimus, Ch. minutus, Ch. varius, Gloeocapsa atrata, Nostoc commune) can colonize, forming small colonies or filaments (Nostoc commune, Phormidium sp. and Oscillatoria sp.) surrounded by gelatinous substances. Apart from cyanobacteria, unicellular algae (Pinnularia borealis, Stichococcus chlorelloides, S. cf. fragilis) together with filamentous algae (Hormidiopsis crenulata, Cylindrocapsa sp., Ulothrix sp.) can also cover the sand surface. The species composition of algae of Bledowska Desert was already investigated by Rahmonov and Piatek [6]. Our results confirm the presence of previously noticed species and present new taxa: $N$. commune, Phormidium sp., Oscillatoria sp. and Ulothrix sp. Algae in post-mining areas contaminated with heavy metals in neighboring of investigated areas has been described by some authors [13-15].

Cyanobacteria in the area studied include Nostoc commune, Phormidium sp. and Oscillatoria sp. These organisms, found in many different climate zones, have the ability to bind free atmospheric nitrogen (except Oscillatoria sp.). Nitrogen accumulation aids biomass increase and microbial association. In arid desert regions, cyanobacteria crusts with Nostoc sp. can successfully stabilize sandy soils as they bind nitrogen [4, 7].

Cyanobacteria and algae most often occur together to form a green coating on the sand surface when there is a lot of moisture, eg in the spring. The algal coating (biofilm), humid in this period, retains all fine organo-mineral particles on their surface. The soil algae stabilize loose ground by binding sand grains which leads to the formation of algal-soil crusts with mineral grains, beginning pedogenesis.

A key feature of soil algae is their ephemeral development, with rapid changes from the dormant stage to active vegetation and vice versa, without any endospores and special intermediate stages $[9,10]$. Surface algal crusts dry out in dry spells and absorb moisture more quickly than dead organic matter. Algae form organic matter that helps to initiate the soil formation process.

Filamentous cyanobacteria and algae play an important role in soil-algal crust formation. On a sand surface, filaments form a complex net covering both individual sand grains and aggregates (Fig. 3 a, c, Fig. 4b) and in which fine-grained mineral and organic fractions are trapped. In these algal nets also, organic membranes deriving from polymeric extracellular substances excreted by soil algae, have been found elsewhere [7, 20, 21]. Similar polymeric structures partly characterise Bledowska Desert crusts (Fig. 3c, Fig. 4a).

The morphological features and structure of soil-algal crusts are very complex. Biogenic fragments and mineral grains ranging from a few microns to several hundred microns in diameter have an important role in crust structure. Microscopic investigations (SEM) enable the recognition of some of the morphological features of the individual components of these crusts. EDXRA analyses of micro-areas can give indicative chemical compositions of mineral, metalorganic and organic aggregates.

Polysaccharides synthesized and excreted by cyanobacteria cells play an important role in interactions with the environment, particularly at times of low water accessibility. Mazor et al [21] have shown the significance of polysaccharide envelopes in the water management 
of algae and microorganisms in desert regions. Due to these abilities, cyanobacteria influence the rate and development of soil microorganisms.

Johansen and Schubert [10] identified the important role of algae in soil formation in desert regions. Rahmonov et al [16] described their significance in initial soil-formation processes on sandy areas in Poland. The important role of soil-algal crusts and microbiotic or biological soil crusts in the ecosystem processes of arid and semi-arid areas has long been recognized [10, 22, 23]. The same role is played in temperate-climate algal crusts [24]. These crusts reduce wind- and water erosion [7, 9], increase nitrogen availability to the ecosystems through fixation and carbon dynamics $[3,4,10]$ and stabilize soil moisture and water relations as in soil crusts [23]. One of the chief factors regulating photosynthesis in cryptobiotic crusts is their moisture content [11]. These crusts may play an important role in mineral erosion and desert soil formation by modifying the weathering environment and ultimately affecting mineralogical variance [8].

Soil algae create conditions suitable for the development of organisms with higher ecological requirements. They indirectly initiate soil formation through cooperation between different soil organisms. Algae also play an important role in areas changed anthropogenically by stimulating soil formation and increasing the potential for plant development $[6,11,16]$. By their development in soils polluted by heavy metals, soil crusts favours ecosystem biodiversity which, in turn, serves to accelerate soil-algal crust formation $[5,6]$.

In Bledowska Desert, the eolian transport is one of the factors limiting the development of biocenotic systems and inhibiting soil formation. Winds dry out mineral material. Sand grains on a surface ground are rounded. Microfissures and hollows as seen in SEM pictures are formed.

An soil-algal crust is a source of mineral and organic compounds that originated from in situ and ex situ. That compounds contain micro and macroelements in concentrations that depend on the type of surrounding landscapes. Nutrients can be taken by both lower- and higher vegetation, and by encroaching vascular plants.

Most algae excrete organic extracellular compounds which enable the binding of exchangeable cations in soils, forming chelates $[18,19]$. This leads to a positive correlation between organic-matter content and basic cations in soils below algocenoses.

Values of organic carbon content $(0.35-2.23 \%)$ and total nitrogen $(0.014-0.113 \%)$ indicate a large mineral fraction in soil crusts. In the algal-soil crust investigated here, there are enrichments of $\mathrm{K}, \mathrm{Ca}, \mathrm{Mg}$ and, to the lesser degree, of $\mathrm{Na}$ and $\mathrm{Mn}$. Rich in various elements grains accumulate in algal net and can improve fertility of poor sandy soils, thus contributing to an increase of different ecological niche.

Accumulation of heavy metals by soil-algal crusts seems to be particularly significant even if the humus horizon (thickness $<5 \mathrm{~mm}$ ) is in an initial stage of development. Heavy metals concentrations are at background level, those of zinc and copper are higher in the Bledowska Desert crusts than in the underlying sands. Elevated concentrations of cadmium also occur in the crusts and lead concentrations are much higher than the background.

The pyrolytical analyses of the soil-algal crust matter show that products of polysaccharide origin dominate in pyrolysates, and that those derived from proteins, lipids and phenols are minor. The forming ectoorganic horizon contains only traces of aromatic compounds and phenol derivatives, probably reflecting the allochtonic input of vascular plant remains. Literature data show that aromatic compounds in algal biomass derive from 
lignin related to vascular plants $[25,26]$. Similar data for sandy areas with soil-algal crusts are given by Nierop et al [19].

From the point of view of vegetation development and of their influence on soil formation, nutrients, particularly nitrogen, are vital. Nitrogen, a deficiency element in poor ecosystems, decreases the rate of soil development. Our research shows that, in the organic matter building the algal crust, nitrogen-bearing compounds are important. Nitrogen, a basic nutrient of plants, generally does not come from weathered soil minerals but is of organic origin. Some of these come from their biological precursors including proteins, chlorophyll, nucleic acids and alcaloids introduced into soils systems as plant- and animal remains [26]. Greater amounts of nitrogen compounds may be related to the occurrence of microorganisms (mainly bacteria) and the products of their metabolism.

The abundance of organic compounds in soil below algal communities results from the fact that these organisms excrete gelatinous (mucus) substances which sustain moisture and indirectly help in soil microflora development. This exudate contains carbohydrates, lipids and other organic compounds which promote the activity of other microorganisms [7, 21]. Transformation and decomposition of algae biomass contribute to the rapid accumulation of organic matter in the soil, which is a potential source of nutrients for early succession and late succession species [27]. Organic matter leads to the pedogenesis in poor sandy areas and initiates the formation of primary humus.

\section{Conclusions}

In the Bledowska Desert, algae are pioneering organisms colonizing areas that lack a soil cover. The formation of soil-algal crusts creates good conditions for later soil development as it promotes organic matter production, microorganisms and fungi association. In regions exposed to pollution, algal crusts trap contaminants from atmospheric emissions. Due to algal crusts, mineral grains of various chemical compositions, accompanied by biotic grains, spores and pollens, microorganisms, particles of cellulose and lignin, are accumulated in soil. Such a structurally and chemically variable substratum offers better conditions for water retention and the later development of organic soil.

Soil organic matter variably depends on algal biomass and on the dynamics of their development. Derivative carbohydrates and nitrogen containing compounds are common in the pyrolytical products of organic matter in soil-algal crust. Nitrogen, a deficiency element in poor ecosystems, decreases the rate of soil development.

The initial organic matter in soil mainly comes from algal biomass. Among organic compounds having the important influence on soil development are carbohydrate derivatives, nitrogen-, sulphur- and oxygen compounds such as pyrane, and furane derivatives. Rapid decay and humification of algal biomasses enables the relatively quick accumulation of organic matter in soil which becomes a source of nutrients for early and later succession species with higher habitat requirements.

\section{Acknowledgments}

The authors gratefully thank the anonymous reviewers for their critical and constructive comments. We also thank Jolanta Piątek for valuable advices and helpful remarks 
concerning algae materials. We thank Dr. Padhraig S. Kennan (University College Dublin, Ireland) for his help in improving the English of this paper.

\section{References}

[1] Fisher T, Veste M, Schaaf W, Dumüg A, Kögel-Knabner I, Wiehe W, et al. Initial pedogenesis in a topsoil crust 3 years after construction of an artificial catchment in Brandenburg, NE Germany. Biogeochemistry. 2010;101:165-176. DOI: 10.1007/s10533-010-9464-Z.

[2] Lukešová A. Soil algae in brown coal and lignite post-mining areas in Central Europe (Czech Republic and Germany). Restor Ecol. 2001;9:341-350. DOI: 10.1046/j.1526-100X.2001.94002.x.

[3] Evans RD, Lange OL. Biological soil crusts and ecosystem nitrogen and carbon dynamics. Ecol Stud. 2003;150:263-279. DOI: 10.1007/978-3-642-56475-8_20.

[4] Belnap J. Factor influencing nitrogen fixation and nitrogen release in biological soil crusts. Ecol Stud. 2003;150:241-261. DOI: 10.1007/978-3-642-56475-8_19.

[5] Pluis JLA. Algal crust formation in the inland dune area. Laarder Wasmeer, the Netherlands. Vegetatio. 1994;113:41-51. DOI: 10.1007/BF00045462.

[6] Rahmonov O, Piątek J. Sand colonization and initiation of soil development by cyanobacteria and algae. Ekológia (Bratislava). 2007;26(1):52-63. http://147.213.211.222/sites/default/files/ Ekol_10705_rahmonov.pdf.

[7] Malam Io, Le Bissonnais Y, Défarge C, Trichet J. Role of a cyanobacterial cover on structural stability of sandy soils in the Sahelian part of western Niger. Geoderma. 2001;101:15-30. DOI: 10.1016/S0016-7061(00)00093-8.

[8] Chen R, Zhang Y, Li Y, Wie W, Zhang J, Wu N. The variation of morphological features and mineralogical components of biological soil crusts in the Gurbantunggut Desert of Northwestern China. Environ Geol. 2009;57:1135-1143. DOI: 10.1007/s00254-008-1410-1.

[9] Johansen JR, Shubert LE. Algae in soil. Nova Hedwigia, Beih. 2001;123:297-306.

[10] Starks TL, Shubert LE. Colonization and succession of algae and soil algae interactions associated with disturbed areas. J Phycol. 1982;18:99-107. DOI: 10.1111/j.1529-8817.1982.tb03162.x.

[11] Belnap J, Büdel B, Lange OL. Biological soil crust: characteristics and distribution. Ecol Stud. 2003;150:3-30. DOI: 10.1007/978-3-642-56475-8_1.

[12] Picińska-Fałtynowicz J. Algae. In: Piotrowska H, editor. The Nature of Słowiński National Park. Poznań-Gdańsk: Bogucki Science Press; 1997.

[13] Kalinowska R, Trzcińska M, Pawlik-Skowrońska B. Glony glebowe terenów pogórniczych skażonych metalami ciężkimi (Soil alga in post-mining areas contaminated with heavy metals). Wiadomości Botaniczne. 2008;52(3/4):63-79.

[14] Kalinowska R, Pawlik-Skowrońska B. Metal resistance of soil algae (Chlorophyta) occurring in post flotation $\mathrm{Zn} / \mathrm{Pb}$ - and Cu-tailing ponds. Pol J Ecol. 2008;56(3):415-430.

[15] Trzcińska M, Pawlik-Skowrońska B. Soil algal communities inhabiting zinc and lead mine spoils. J Appl Phycol. 2008;20:341-348. DOI: 10.1007/s10811-007-9259-3.

[16] Rahmonov O, Kowalski WJ, Bednarek R. Characterization of the soil organic matter and plant tissues in an initial stage of plant succession and soil development by means of Curie-point pyrolysis coupled with GC-MS. Eurasian Soil Sci. 2010;43(13):1557-1568. DOI: 10.1134/S1064229310130144.

[17] Guiry MD, Guiry GM. AlgaeBase. World-wide electronic publication. Galway: National University of Ireland; 2013. http://www.algaebase.org.

[18] Chefetz B, Tarchitzky J, Deshmukh AP, Hatcher PG, Chen Y. Structural characterization of soil organic matter and humic acids in particle-size fractions of an agricultural soil. Soil Sci Soc Am J. 2002;66:129-141. DOI: 10.2136/sssaj2002.1290.

[19] Nierop KGJ, Van Lagen B, Buurman P. Composition of plant tissues and soil organic matter in the first stages of a vegetation succession. Geoderma. 2001;100:1-24. DOI: 10.1016/S0016-7061(00)00078-1.

[20] Lechowski Z, Białczak J. Rola i znaczenie polisacharydów sinic w przyrodzie i biotechnologii (Role of cyanobacterial polysaccharides in environment and biotechnology). Wiadomości Botaniczne. 2001;45(3/4):35-51. http://bomax.botany.pl/pubs/data/article_pdf?id=2325.

[21] Mazor G. Kidron GJ, Vonshak A, Abeliovich A. The role of cyanobacterial exopolysaccharides in structuring desert microbial crusts. FEMS Microbiol Ecol. 1996;21(2):121-130. DOI: 10.1016/0168-6496(96)00050-5. 
[22] Dümig A, Veste M, Hagedorn F, Fischer T, Lange P, Spröte R, et al. Biological soil crusts on initial soils: organic carbon dynamics and chemistry under temperate climatic conditions. Biogeosci Discuss. 2013;10:851-894. DOI: 10.5194/bgd-10-851-2013.

[23] Eldridge DJ. Biological soil crusts and water relations in Australian Deserts. Ecological Studies. 2003;150:315-325. DOI: 10.1007/978-3-642-56475-8_23.

[24] Shtina EA. The peculiarities of algal flora in the anthropogenic soil (by the example of Valaam Island). Eurasian Soil Sci. 2000;33(8):847-849.

[25] Page DW, Van Leeuwen AJ, Spark KM, Mulcahy DE. Pyrolysis characterization of plant, humus and soil extract from Australian catchments. J Anal Appl Pyrol. 2002;65:269-285. DOI: 10.1016/S0165-2370(02)00005-0.

[26] Schulten HR, Schnitzer M. The chemistry of soil organic nitrogen: a review. Biol Fert Soils. 1998;26:1-15. DOI: $10.1007 / \mathrm{s} 003740050335$.

[27] Rahmonov O, Gajos M, Czuban R, Parusel T. GIS methods in monitoring succession processes in limestone and dolomite quarries. Pol J Environ Stud. 2014;23(2):647-653. http://www.researchgate.net/publication/ 261850855_GIS_Methods_in_Monitoring_Succession_Processes_in_Limestone_and_Dolomite_Quarries.

\title{
ROLA GLONÓW GLEBOWYCH W INICJALNYCH ETAPACH ROZWOJU GLEBY NA ZANIECZYSZCZONYCH OBSZARACH PIASZCZYSTYCH
}

\author{
${ }^{1}$ Wydział Nauk o Ziemi, Uniwersytet Śląski, Sosnowiec \\ ${ }^{2}$ Wydział Nauk o Ziemi, Uniwersytet Mikołaja Kopernika, Toruń \\ ${ }^{3}$ Wydział Technologii Żywności, Uniwersytet Rolniczy im. Hugona Kołłątaja, Kraków
}

\begin{abstract}
Abstrakt: Przeprowadzone badania miały na celu określenie roli glonów glebowych w inicjalnym stadium rozwoju gleb na obszarach piaszczystych oraz rozpoznanie składu mineralnego i chemicznego skorup glonowo-glebowych. Obszar badań leży w południowej Polsce na terenie ruchomych piasków (Pustynia Błędowska), powstałych w wyniku wycinki lasów przeprowadzonej na potrzeby średniowiecznego górnictwa $\mathrm{Ag}-\mathrm{Pb}-\mathrm{Fe}$. Zidentyfikowano piętnaście gatunków glonów (Cyanophyta, Chlorophyta, Heterokontophyta). W skorupach glonowo-glebowych zawartość $\mathrm{C}_{\text {org }}$ wahała się w granicach 0,35-2,23\%, $\mathrm{P}_{\text {przyswajalny }}$ - 9-34 $\mathrm{mg} \cdot \mathrm{kg}^{-1}$, natomiast zawartość Nt była zróżnicowana. Na obszarze badań podłoże, na którym rozwijały się glony i sinice, było kwaśne ( $\mathrm{pH}$ 4,4-5,7 w $\mathrm{KCl}$ ). Wśród składników mineralnych występujących w skorupach glonowo-glebowych dominowały fazy bogate w Fe i K (odpowiednio: 776,5( $\pm 2,3)-2803( \pm 31) \mathrm{mg} \cdot \mathrm{kg}^{-1}$ i 230,5( $\left.\pm 3,7)-696( \pm 22) \mathrm{mg} \cdot \mathrm{kg}^{-1}\right)$. Zaobserwowano w nich również wysoką koncentrację $\mathrm{Zn}, \mathrm{Mn}$ i Pb. Materia organiczna badanych skorup zawierała związki alifatyczne i aromatyczne, pochodne węglowodanów, fenole, furan i piren. Znaczący udział w składzie skorup glonowo-glebowych miały też związki azotu - glony są głównym źródłem materii organicznej w badanym stadium formowania się gleby, co może się przyczyniać do szybkiej regeneracji odkształconych układów ekologicznych.
\end{abstract}

Słowa kluczowe: metale ciężkie, związki organiczne, glony glebowe, skorupa glonowo-glebowa, rozwój gleby 